\title{
Hidden Markov Models for Activity Detection in Atrial Fibrillation Electrograms
}

\author{
Gonzalo R Ríos-Muñoz ${ }^{1,2,3,4 *}$, Fernando Moreno-Pino ${ }^{1,4}$, Nina Soto ${ }^{2}$, Pablo M Olmos ${ }^{1,4}$, Antonio \\ Artés-Rodríguez ${ }^{1,4}$, Francisco Fernández-Avilés ${ }^{1,2,3,5,6}$, Ángel Arenal ${ }^{1,5}$ \\ ${ }^{1}$ Instituto de Investigación Sanitaria Gregorio Marañón (IiSGM), Madrid, Spain ${ }^{2}$ Hospital General \\ Universitario Gregorio Marañón, Madrid, Spain ${ }^{3}$ Red de Terapia Celular (TERCEL), Instituto de \\ Salud Carlos III, Madrid, Spain ${ }^{4}$ Universidad Carlos III de Madrid, Spain ${ }^{5}$ Spanish Network Centre \\ on Cardiovascular Research (CiberCV), Spain ${ }^{6}$ Universidad Complutense de Madrid, Spain
}

\begin{abstract}
Activity detection in atrial fibrillation (AF) electrograms (EGMs) is a key concept to understand the mechanisms of this frequent arrhythmia and design new strategies for its treatment. We present a new method that employs Hidden Markov Models (HMMs) to identify activity presence in bipolar EGMs. The method is fully unsupervised and hence it does not require labeled training data. The HMM activity detection method was validated and compared to the non-linear energy operator (NLEO) method for a set of manually annotated EGMs. The HMM performed better than the NLEO and exhibited more robustness in the presence of low voltage fragmented EGMs.
\end{abstract}

\section{Introduction}

Fragmentation presence in atrial fibrillation (AF) electrograms (EGMs) has been extensively studied in the literature, with areas exhibiting complex fractionated atrial electrograms (CFAEs) postulated as responsible for $\mathrm{AF}$ maintenance [1]. However, the algorithms applied to detect CFAEs are very dependent of the specific settings and parameters used by the different operators.

Complementing CFAEs, the Activity Ratio (AR) index was first introduced in [2]. By first applying a signal processing algorithm the method obtains a binary signal that classifies the EGM into active or inactive. It relies on the non-linear energy operator (NLEO) [3], that relates the energy of a signal to the activity. The NLEO method has been used to identify local activation times (LATs) that represent the real activation of the tissue in contact with the electrode [4]. However, its adaptive threshold entails further validation of its parameters. Additionally, the filtering steps applied might distort the signal, diminishing low voltage signals associated to fragmented EGMs, which can be miss-detected as inactive sections.
To improve the detection of fragmentation in atrial EGMs, we propose an unsupervised approach based on Hidden Markov Models (HMMs) [5]. Probabilistic generative model that approximates the true distribution of the data using a set of hidden states and can hence describe the evolution of observable events, i.e., EGMs, that depend on inner hidden factors that cannot be observed. Our objective is to train a HMM that can quantify the presence of activity in a bipolar atrial AF EGM. The HMMs can operate in the absence of training labeled data, making the whole training process unsupervised. Hence, no expert annotation is needed for training and only EGM signals are required.

\section{Materials}

A total of 3780 AF bipolar EGMs of 2500 ms length were acquired from 32 persistent $\mathrm{AF}$ patients using a 5 branch PentaRay catheter. All patients gave full informed consent and the study was approved by the Institutional Review Board of the center. The sampling frequency was $f_{s}=1 \mathrm{kHz}$, signals were band-pass filtered $30-240 \mathrm{~Hz}$. Signals exhibited different characteristics such as discrete activations, fragmentation, double potentials or no signal (noise). To validate the algorithm and compare it to the NLEO method, the activity of 232 signals was manually labeled by two electrophysiologists for activity presence, 0 for no activity and 1 for activity.

The HMM code employed in the study is available in [6]. The hardware specifications of the system were: one 8-core Intel ${ }^{\circledR} \mathrm{Xeon}^{\circledR} \mathrm{CPU} 3.40 \mathrm{GHz}$ processor, $16 \mathrm{~GB}$ RAM, on 64 bits Windows 7 Professional. A Titan V GPU was also used to validate and test the methods.

\section{Methods}

In this section, we provide a brief introduction to HMMs, model training, and the NLEO method used to 
compare the EGM activity detection performance on the labeled database.

\subsection{Hidden Markov Model}

A Hidden Markov Model or HMM consists of a discrete-time, discrete-state Markov chain, with hidden states $S=\left\{s_{1}, s_{2}, \ldots, s_{T}: s_{t} \in 1, \ldots, H\right\}$, where $H$ is the number of hidden states and $T$ the number of observation samples, plus an observation model $p\left(y_{t} \mid s_{t}\right)$, where the observed sequence is defined by $Y=$ $\left\{y_{1}, y_{2}, \ldots, y_{T}: y_{t} \in \mathbb{R}^{d}\right\}$ [7], and $d$ is the data dimension. Figure 1 shows the HMM block diagram.

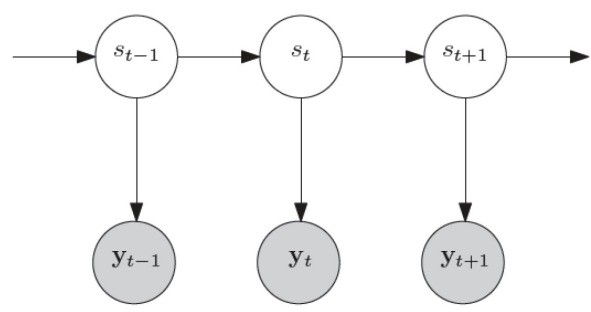

Figure 1. Hidden Markov Model block diagram.

The parameters of the model $\theta=\{\mathbf{A}, \mathbf{B}, \boldsymbol{\pi}\}$ that define a HMM are [2]:

- $\mathbf{A}=\left\{\mathbf{a}_{\mathbf{i j}}: \mathbf{a}_{\mathbf{i j}}=\mathbf{p}\left(\mathbf{s}_{\mathbf{t}+\mathbf{1}}=\mathbf{j} \mid \mathbf{s}_{\mathbf{t}}=\mathbf{i}\right)\right\}$, the state transition probabilities, i.e., the probability of changing from one state to another for the next time instant.

- $\mathbf{B}=\left\{\mathbf{b}_{\mathbf{i}}: \mathbf{p}_{\mathbf{b}_{\mathbf{i}}}\left(\mathbf{y}_{\mathbf{t}}\right)=\mathbf{p}\left(\mathbf{y}_{\mathbf{t}} \mid \mathbf{s}_{\mathbf{t}}=\mathbf{i}\right)\right\}$, the observation emission probabilities associated to each state. For our model, the observations are real-valued continuous observations and hence a Gaussian conditional distribution $p(y \mid s)$ is assumed [5].

- $\boldsymbol{\pi}=\left\{\pi_{i}: \pi_{i}=p\left(s_{1}=i\right)\right\}$, the initial state probability distribution. In our model they are initially set according to a K-Means clustering algorithm applied to the observations for a number of clusters equals the number of HMM states $H$ to accelerate the convergence to a solution.

In order to train the HMMs for EGM activity detection we must solve three basic problems of interest:

1. Calculate $p(Y \mid \theta)$, the probability of the observations $Y=\left\{y_{1}, y_{2}, \ldots, y_{T}\right\}$ given the model $\theta=\{\mathbf{A}, \mathbf{B}, \boldsymbol{\pi}\}$.

2. Compute $S=\left\{s_{1}, s_{2}, \ldots, s_{T}: s_{t}\right\}$, the optimal state sequence that best "explains" the observations and that maximizes $p(S \mid Y, \theta)$ given the observed sequence $Y$ and the model parameters $\theta$.

3. Calculate the optimal model parameters $\theta$ that maximize $p(Y \mid \theta)$.

The solutions for these problems are well known [5, 7]. To solve the first problem we used the Forward algorithm to compute $p(Y \mid \theta)$. For the second problem, we employed the Viterbi algorithm, that optimizes the $\underset{S}{\operatorname{argmax}} p(S \mid Y, \theta)$. Alternatively, the Forward-Backward S algorithm can also be implemented $[5,7]$. To solve the third problem we applied the Expectation-Maximization (EM) method, also known as Baum-Welch algorithm. In conclusion, given the number of hidden states $H$ and observations $Y=\left\{y_{1}, y_{2}, \ldots, y_{T}\right\}$, we first compute the optimal $\theta$ that will allow us to later find the optimal hidden state sequence that better explains our observations.

\subsection{HMM for EGM Activity Detection}

The input parameters to train the models were the number of hidden states $H$, the number and length of the EGMs (sequences), $N$ and $T$ respectively. No pre-processing was applied to the signals. The dimension of the observations in our application was $d=1$. We applied the methods in the previous solutions to train different HMMs for different number of signals and durations, see Table 1. Further details can be found in the Simulations section.

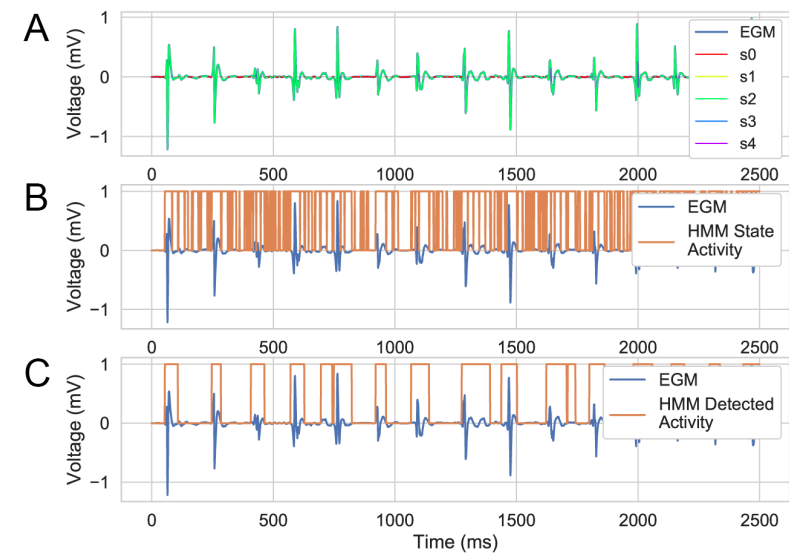

Figure 2. HMM EGM activity detection steps. A: Bipolar EGM decoded sequence for $H=5$ states. B: Activity related to the states. C: HMM activity detection result.

Table 1. HMM trained models parameters.

\begin{tabular}{cll}
\hline \hline Parameter & Description & Range \\
\hline$H$ & $\begin{array}{l}\text { Number of HMM } \\
\text { states }\end{array}$ & {$[2: 1: 10]$} \\
$N$ & $\begin{array}{l}\text { Number of training } \\
\text { EGMs }\end{array}$ & {$[10,50,100,250,500]$} \\
& EGMs length (ms) & {$[250,500: 500: 2500]$} \\
\hline & Total Trained Models & 270 \\
\hline
\end{tabular}

Once the model is trained, i.e., the $\theta=\{\mathbf{A}, \mathbf{B}, \boldsymbol{\pi}\}$ that best describe the observations is obtained, we apply the following steps to detect the activity on the EGMs:

1. Decoding HMM States: Bipolar signals are directly fed to the HMM model. The most likely states sequence associated to the EGM is calculated. See Figure 2A. 
2. HMM State Activity: The state with the minimum energy associated to its decoded EGM observations is automatically considered to represent no activity. The rest of the states are clustered and associated to EGM activity. Therefore a binary signal is obtained taking value 0 when the signal remains in the inactive state and value 1 for the active states, as shown in Figure 2B.

3. Postprocessing: Similarly to the NLEO method, inactive segments shorter than a refractory period $M$ are set to 'active', merging neighboring sections. Active sections shorter than $D$ are discarded, which removes outliers and voltage spikes, as Figure $2 \mathrm{C}$ shows.

\subsection{NLEO for Activity Detection}

The NLEO algorithm was chosen to compare the proposed method. The NLEO applies a sliding window operation to the discretized EGM voltage signal $x[n]$ as follows:

$$
N L E O[n]=x^{2}[n]-x[n+1] \cdot x[n-1] .
$$

The complete signal processing steps to detect the activity of a bipolar EGM is described in [8]. It consists of

1. Denoising and Baseline Wander Removal.

2. Apply the NLEO operator.

3. Gaussian Lowpass Filtering.

4. Adaptive thresholding to obtain a binary activity signal.

5. Postprocessing to merge close active sections and discard short activations.

\subsection{Simulations}

Tables 1, 2 and 3 include all the simulated parameters and their values for the methods. For the NLEO method, we set different values for the Gaussian window size $G$, the post-processing merge distance $M$, the discard duration $D$, and the adaptive threshold value $V$. For each of the 270 trained HMMs from the simulation parameters in Table 1, we applied the same post-processing merge distance $M$ and discard duration $D$ variables. We ran a total of 122210 simulations for the NLEO and 5670 for the HMMs.

Table 2. NLEO validation parameters.

\begin{tabular}{clc}
\hline \hline Parameter & Description & Range \\
\hline$W$ & Gaussian window size $(\mathrm{ms})$ & {$[10: 10: 100]$} \\
$M$ & Merge distance $(\mathrm{ms})$ & {$[0: 5: 50]$} \\
$D$ & Discard duration $(\mathrm{ms})$ & {$[0: 1: 10]$} \\
$V$ & Threshold values & {$[0: 0.01: 1]$} \\
\hline & Total Simulations & 122210 \\
\hline
\end{tabular}

Table 3. Validation parameters for each trained HMM model.

\begin{tabular}{clc}
\hline \hline Parameter & Description & Range \\
\hline$M$ & Merge distance $(\mathrm{ms})$ & {$[0: 5: 50]$} \\
$D$ & Discard duration $(\mathrm{ms})$ & {$[0: 1: 10]$} \\
\hline \multicolumn{2}{c}{ Total Simulations per Trained Model } & 21 \\
\hline Total Simulations for the 270 Trained Models & 5670 \\
\hline \hline
\end{tabular}

\section{Results}

\subsection{HMM Evaluation}

The HMM activity detection is based on unsupervised learning. To evaluate the trained models and confirm they are learning from the data, we calculated two probabilistic model selection criteria: the Akaike Information Criterion (AIC), and the Bayesian Information Criterion (BIC) [9]. They take the number of states of the models, and the trainable parameters and analyze their complexity as follows:

$$
\begin{gathered}
A I C=2 \cdot P-2 \cdot \mathcal{L}\left(\theta \mid y_{1}, \cdots, y_{N}\right), \\
B I C=P \cdot \log (T)-2 \cdot \mathcal{L}\left(\theta \mid y_{1}, \cdots, y_{N}\right),
\end{gathered}
$$

where $P$ is the number of trainable parameters of the model $\theta, \mathcal{L}\left(\theta \mid y_{1}, \cdots, y_{N}\right)$ the log-likelihood of the observed data for model $\theta$, and $T$ the length of the signals.

In Figure 3 we show the AIC and BIC metrics of a trained model for $N=100$ signals and $T=250 \mathrm{~ms}$. AIC and BIC perform similarly, and we appreciate that the optimal value of states is $H=5$ or $H=6$, with complexity increasing for higher number of states.

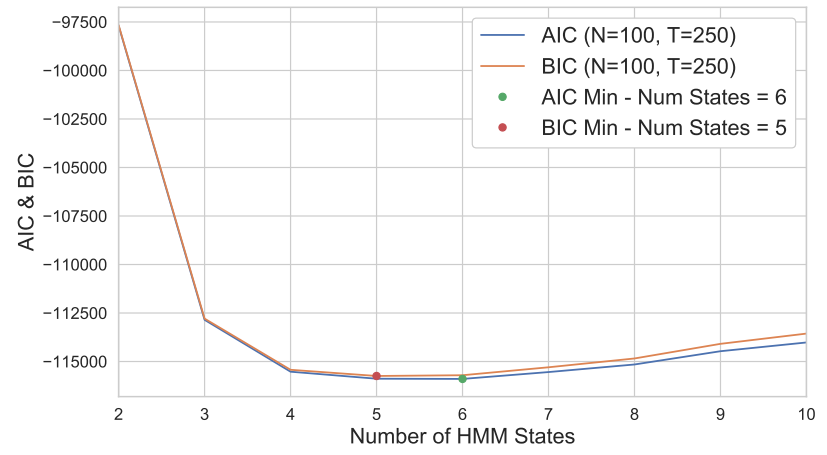

Figure 3. AIC/BIC analysis of a trained HMM. Training parameters $N=100$ signals and $T=250 \mathrm{~ms}$.

\subsection{Activity Detection Results}

The F1score for activity detection was calculated to compare NLEO and HMM methods. We computed the F1Score for all the 122210 different NLEO and 5670 
HMM simulations for the 232 manually labeled AF EGMs. The parameter combinations for the best performance on both models is included in Table 4. The HMM method performed better than the NLEO in terms of F1Score, 0.960 versus 0.902 respectively. In Figure 4 we include an example of activity detection on different EGMs using the best parameters of both methods and the manual annotations.
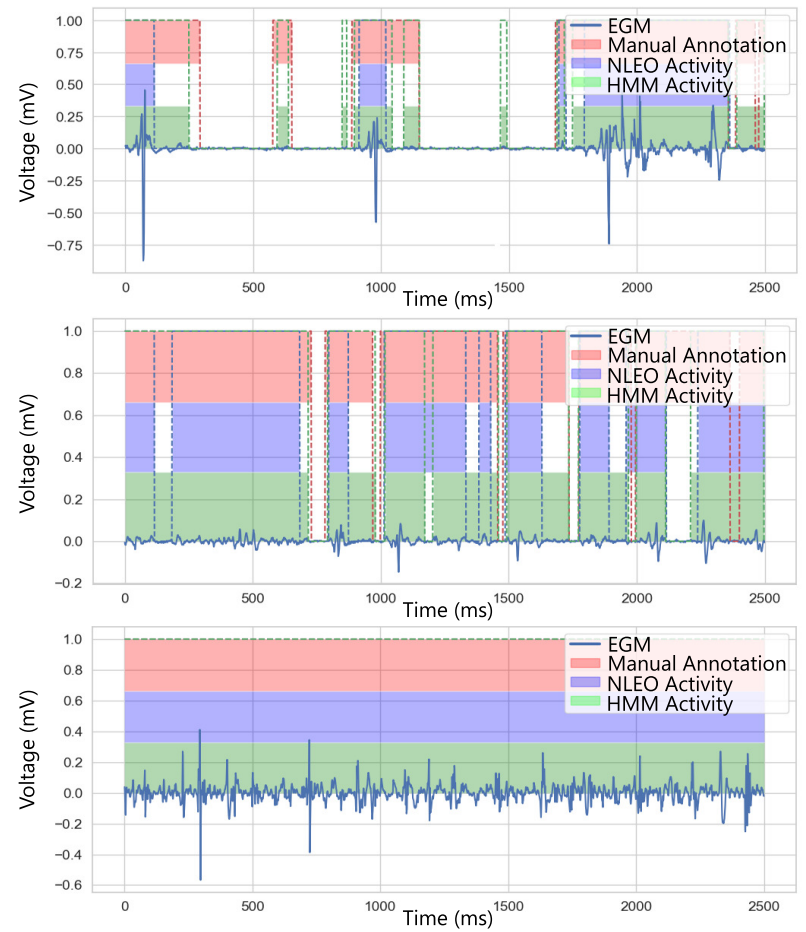

Figure 4. NLEO and HMM EGM activity detection methods compared to the manually annotated signals.

Table 4. Best F1Score for the NLEO and HMM EGM activity detection methods on the manually annotated EGMs.

\begin{tabular}{llc}
\hline \hline Method & Parameters & F1Score \\
\hline NLEO & $\mathrm{W}=70, \mathrm{M}=50, \mathrm{D}=0, \mathrm{~V}=0$ & 0.902 \\
HMM & $\mathrm{N}=50, \mathrm{~T}=500, \mathrm{H}=5, \mathrm{M}=30, \mathrm{D}=9$ & $\mathbf{0 . 9 6 0}$ \\
\hline \hline
\end{tabular}

\section{Conclusions}

We have presented a new algorithm that detects EGM activity in AF signals based on HMMs. The HMM learning was fully unsupervised, with no expert annotation involved in the training. Additionally, the method does not require pre-processing of the EGMs, which avoids signal manipulation and distortion caused by other filtering pre-processing methods. We have compared our method to the NLEO for activity detection on a manually labeled database. Our method performed better and demonstrated to be more robust regarding different voltage amplitudes of the signals, it can operate automatically to detect EGM activity and requires less parameters involved in the calculation. As future works, the HMM method can be applied to electro-anatomical maps to assess the presence of fragmented areas in the atria of the patients and can serve as prior step to narrow and detect local activation times in $\mathrm{AF}$ EGMs.

\section{Acknowledgments}

This study was supported by grants PI18/01895 from the Instituto de Salud Carlos III, and RD16/0011/0029 Red de Terapia Celular from the Instituto de Salud Carlos III, the projects RTI2018-099655-B-I00; TEC2017-92552-EXP; PID2019-108539RB-C22, Y2018/TCS-4705, and the support of NVIDIA Corporation with the donation of the Titan V GPU used during this research.

\section{References}

[1] Nademanee K, et al. A new approach for catheter ablation of atrial fibrillation: Mapping of the electrophysiologic substrate. Journal of the American College of Cardiology 2004; 43(11):2044-53.

[2] Schilling C, et al. Non-linear energy operator for the analysis of intracardial electrograms. volume 25-4 of IFMBE Proceedings. 2009; $872-875$.

[3] Kaiser JF. On a simple algorithm to calculate the 'energy' of a signal. In ICASSP, IEEE International Conference on Acoustics, Speech and Signal Processing - Proceedings, volume 1. Publ by IEEE, 1990; 381-384.

[4] Burdumy M, et al. Comparing measured and simulated wave directions in the left atrium a workflow for model personalization and validation. Biomedizinische Technik 2012; 57(2):79-87.

[5] Rabiner LR. A tutorial on hidden markov models and selected applications in speech recognition. Proceedings of the IEEE 1989;77(2):257-286.

[6] Moreno-Pino F, Sukei E, Artés-Rodríguez A. fmorenopino/heterogeneoushmm: First stable release of heterogenoushmm. https://github.com/ fmorenopino/Heterogeneous HMM, apr 2020.

[7] Murphy KP. Machine learning : a probabilistic perspective. Cambridge, Mass. [u.a.]: MIT Press, 2013.

[8] Nguyen MP, Schilling C, Dössel O. A new approach for automated location of active segments in intracardiac electrograms. In World Congress on Medical Physics and Biomedical Engineering, 2009. 2010; 763-766.

[9] Burnham KP, Anderson DR. Multimodel inference understanding aic and bic in model selection. Sociological Methods and Reserarch 2004;33(2):261-304.

Address for correspondence:

Gonzalo R. Ríos-Muñoz

Instituto de Investigación Sanitaria Gregorio Marañón

Calle Doctor Esquerdo 46 28028, Madrid, Spain

griosm@tsc.uc3m.es 